A N N A L E S Annales de Bretagne et des Pays de l'Ouest

Anjou. Maine. Poitou-Charente. Touraine

111-3 | 2004

Alcuin de York à Tours

\title{
Alcuin et les doctrines grammaticales
}

\section{Pierre Swiggers}

\section{OpenEdition}

Journals

Édition électronique

URL : http://journals.openedition.org/abpo/1231

DOI : $10.4000 / a b p o .1231$

ISBN : 978-2-7535-1495-9

ISSN : 2108-6443

Éditeur

Presses universitaires de Rennes

Édition imprimée

Date de publication : 20 septembre 2004

Pagination : 147-161

ISBN : 978-2-7535-0053-2

ISSN : 0399-0826

Référence électronique

Pierre Swiggers, "Alcuin et les doctrines grammaticales », Annales de Bretagne et des Pays de l'Ouest [En ligne], 111-3 | 2004, mis en ligne le 20 septembre 2006, consulté le 21 avril 2019. URL : http:// journals.openedition.org/abpo/1231; DOI : 10.4000/abpo.1231 


\title{
Alcuin et les doctrines grammaticales
}

\author{
Pierre SWIGGERS \\ Professeur au Département de Linguistique \\ Centre international de dialectologie générale, K.U. Leuven
}

Nous nous intéresserons ici à Alcuin grammaticus, c'est-à-dire Alcuin comme lecteur de grammaires antiques, comme grammairien ayant réfléchi aux structures de la langue latine et comme didacticien de la grammaire. Cette triple dimension est présente dans l'œuvre qu'on désigne comme ars grammatica, ou encore comme de grammatica (et partibus orationis et eorum accidentibus per dialogum), de octo partibus orationis, dialogus Saxonis et Franconis de octo partibus orationis, voire comme enchiridion sive grammatica in modum dialogi inter Francum [Franconem] et Saxonem, ou grammaticae breviarium.

Ce texte, conservé dans de nombreux manuscrits ${ }^{1}$ du IX ${ }^{\mathrm{e}}$ siècle (Angers, Berne, Florence, Laon, Mersebourg, Munich, Naples, Paris, Saint-Gall, Trèves et Vatican), n'a pas encore fait l'objet d'une édition critique ${ }^{2}$. Il a sans doute été entamé et préparé (en grande partie) à l'époque où Alcuin résidait à la Cour de Charlemagne; on peut supposer qu'il a été terminé à Tours ${ }^{3}$, où Alcuin avait fait venir un certain nombre d'ouvrages indispensables pour rédiger une synthèse du " savoir grammatical de base ". Car son ars grammatica est bien une telle synthèse, vu que :

1- le savoir grammatical y est présenté comme un champ autonome (distinct de la métrique ${ }^{4}$ et de la rhétorique);

1. Voir la notice dans M.-H. Jullien et F. Perelman, Clavis ..., p. 21-23.

2. Cf. L. Hotrz, " Priscien dans la pédagogie... ", p. 294 : " Ce texte mériterait une édition critique authentique "; une édition critique serait en préparation [par Martin Irvine]. Nous citons le texte d'après l'édition dans PL 101, col. 849-902 (à quelques rares endroits, nous avons corrigé l'édition).

3. On sait que c'est à l'abbaye de Saint-Martin de Tours qu'Alcuin a composé la presque totalité de ses œuvres; mais L. HotTz, "Priscien dans la pédagogie... ", p. 294, a certainement raison de supposer que le dialogue entre Franco et Saxo a été rédigé déjà en partie à la Cour de Charlemagne.

4. Les questions de métrique ne sont pas abordées : Sed has rationes metricae subtilitates esse reor, in quibus necdum eruditi sumus (Sa.) [PL 101, 856]; Sed haec [= syllabae communes], quia ad metricos pertinent, relinquamus (Sa.) [PL 101, 857]; Plenius haec, vita comite, in metrica ratione vobis, filii, monstravero (Mag.) [PL 101, 857]; Amplius haec quoque in illa metrorum subtilitate intelligetis (Mag.) [PL 101, 857]. 
2- l'information fournie vise à mettre en place les notions indispensables pour une bonne compréhension de la nature et de la fonction des structures linguistiques (du latin). En ce sens, l'ouvrage n'est certainement pas à considérer comme une grammaire élémentaire du latin : en fait, les données grammaticales y sont présentées dans le cadre d'une réflexion qui présuppose déjà la maîtrise d'une ars élémentaire (comme celle de Donat).

Dans ce qui suit, on étudiera les aspects suivants :

(a) la construction du discours didactique ${ }^{5}$;

(b) la position " intertextuelle " de cet opuscule,

(b') dans l'œuvre d'Alcuin,

(b") dans son rapport avec ses sources;

(c) les traits caractéristiques de la démarche d'Alcuin;

(d) l'apport scientifique et culturel d'Alcuin.

\section{La construction du discours didactique}

L'ars grammatica d'Alcuin est présenté, dès le début, comme une disputatio $^{6}$. Celle-ci met en scène trois personnages. Les protagonistes sont deux jeunes garçons, Franco et Saxo, frais émoulus d'un cours de grammaire élémentaire; entre les deux, il y a une légère différence d'âge, mais surtout une différence de savoir : Saxo doit instruire son collega ${ }^{7}$. Le choix des noms propres suggère une divergence (de nature/de niveau) d'éducation " libérale " (différence qu'Alcuin avait pu apprécier dans sa réalité concrète, par la confrontation du savoir insulaire avec le savoir continental). Le troisième personnage est le magister Albinus (apostrophé comme magister, domine magister et pater), qui est interpellé à quelques reprises. Le début du texte introduit les trois personnages et précise leur statut (intellectuel et discursif) :

Fuerunt in schola Albini magistri duo pueri, unus Franco, alter Saxo, qui nuperrime spineta grammaticae densitatis irruperunt. Quapropter placuit illis paucas litteralis scientiae regulas memoriae causa per interrogationes et responsiones excerpere.

5. Cette partie a été condensée, vu que la structure du dialogue est analysée en détail par L. HoLTz, ici même (supra p. 133-145).

6. Et primum dicite unde vestram convenientius disputationem esse arbitramini incipiendam? (Mag.) [PL 101, 854]; [suit une réflexion sur les éléments constitutifs d'une disputatio: Unde a voce, cujus causa litterae sunt inventae, inchoandam disputationem constat : vel magis primo omnium interrogandum est, quibus modis constet disputatio? (Mag.); Nam nos nescire confitemur quibus modis constet disputatio (DIS.) [PL 101, 854]; Tria sunt quibus omnis collocutio disputatioque perficitur, res, intellectus, voces (Mag.) [PL 101, 854]; Sed quia disputationis modos dixisti, vocis differentias ut exponas, precamur (DIS.) [PL 101, 854]; Sed longum est hoc et huic nostrae puerili disputationi onerosum edicere (Sa.) [PL 101, 862]; Sed vestram vos intrate disputationem; haec alias (Mag.) [PL 101, 874]. Nous indiquons les rôles discursifs à l'aide des abréviations suivantes : DIS. (= discipuli), Fr. (= Franco), Mag. (= Magister), Sa. (= Saxo).

7. Da, collega, divisionem litterarum (Fr.) [PL 101, 855]. Voir M. RoGER, L'enseignement..., p. 357 : "L'aîné joue le rôle de moniteur ". 
At prior illorum Franco dixit Saxoni : Eia, Saxo, me interrogante responde, quia tu majoris es aetatis. Ego XIV annorum; tu ut reor XV. Ad haec Saxo respondit : Faciam; ita tamen, ut si quid altius sit interrogandum, vel ex philosophica disciplina proferendum, liceat magistrum interrogare.

Ad haec magister : Placet, filii, propositio vestra : et libens annuo vestrae sagacitati. Et primum dicite unde vestram convenientius disputationem esse arbitramini incipiendam? [PL 101, 854]

Au début, le magister est bien présent : c'est lui qui identifie (dans un passage qui résume l'enseignement d'Aristote et qui anticipe sur l'agencement global des traités de modis significandi) le point de départ pour la disputatio, à savoir la littera (et son pendant oral, la vox, qui est la " cause " de l'existence de lettres $)^{8}$; c'est aussi le magister qui donne la définition de la littera et la vraie raison d'être de la division entre voyelles et consonnes.

Ensuite, le maître s'efface pour donner libre jeu à ses deux disciples. Car leur disputatio - per interrogationes et responsiones - est un ludus ${ }^{9}$, non seulement par le jeu des rôles ${ }^{10}$, mais aussi par le fait que l'auteur - dans le but de conférer plus de vivacité au dialogue et de tenir en éveil l'esprit du lecteur - peint nos deux jeunes gens comme des concurrents (en matière de savoir grammatical). D'où des échanges enjoués entre Franco avide d'extraire le surplus de science de Saxo, qui est accusé de livrer à contrecœur des bribes de son savoir. Cette touche ludique scande la disputatio et permet à Alcuin de présenter des matières qui relèvent de l'information plus avancée (ou plus élevée).

Le premier rôle discursif du maître est de fixer le point d'ancrage de la disputatio, d'une part par la définition de la littera et de la vox (et cela en rapport direct avec la nature même de la disputatio) ${ }^{11}$, d'autre part - à la suite de l'entrée en matière - par la définition de la grammaire et par un exposé synoptique de ses divisions. Le premier apport (A) est basé essentiellement sur Boèce, De interpretatione liber I; le second (B) principalement sur Isidore de Séville ${ }^{12}$ :

(A) Tria sunt quibus omnis collocutio disputatioque perficitur, res, intellectus, voces. Res sunt, quae animi ratione percipimus. Intellectus, quibus res ipsas addiscimus. Voces, quibus res intellectas proferimus; cujus causa, ut diximus, litterae inventae sunt [...].

Quatuor sunt differentiae vocis : articulata, inarticulata; litterata, illiterata. Articulata est, quae copulata atque coarctata cum sensu profertur, ut : Arma

8. Res sunt, quae animi ratione percipimus. Intellectus, quibus res ipsas addiscimus. Voces, quibus res intellectas proferimus, cujus causa, ut diximus, litterae inventae sunt (Mag.) [PL $101,854]$.

9. Necessarium esset, sed modum nostri ludi excederet (Sa.) [PL 101, 892].

10. Le magister emploie d'ailleurs deux fois le terme de partes dans ce sens (" rôles "): Unde ad partes vos convertite [PL 101, 857]; Nunc ad partes convertite vos [PL 101, 858].

11. Celle-ci repose sur l'expression, orale/écrite, de contenus noétiques (rationes) à propos de " choses" (res).

12. À propos de la synthèse grammaticale qu'on trouve dans les Etymologiae d'Isidore, voir P. SWigGers, "Isidore de Séville... ". 
virumque cano [...] Inarticulata, quae a nullo sensu profiscitur, ut crepitus, mugitus. Litterata, quae scribi potest; illitterata, quae scribi non potest $[\ldots]$ (Mag.) [PL 101, 854]

Littera est individua, quia sententias in partes, partes in syllabas, syllabas in litteras dividimus. Litterae vero indivisibiles sunt [...] (Mag.) [PL 101, 855] ${ }^{13}$

(B) Grammatica est litteralis scientia, et est custos recte loquendi et scribendi; quae constat natura, ratione, auctoritate, consuetudine. (Mag.) [PL 101, $857]^{14}$

[Grammatica dividitur] In XXVI [species]. In vocem, in litteras, in syllabas, partes, dictiones, orationes, definitiones, pedes, accentus, posituras, notas, orthographiam, analogiam, etymologiam, glossas, differentias, barbarismum, soloecismum, vitia, metaplasmum, schemata, tropos, prosam, metra, fabulas, historias. (Mag.) [PL 101, 858]

Cette dernière liste ${ }^{15}$ est presque identique à celle qu'on trouve chez Isidore de Séville, qui toutefois reconnaît les 8 parties du discours comme autant de species, mais qui ne mentionne pas la dictio (qu'Alcuin définit d'après Priscien), ni l'oratio (également définie d'après Priscien), ni la definitio (qu'Isidore traite dans son livre sur la dialectica). Là où Isidore dénombre 30 species, Alcuin en retient 26 (mais si l'on comptait les partes pour 8 espèces, il y en aurait 33) [voir Annexe I].

Directement après, le magister est amené à dépasser le cadre d'un manuel élémentaire ${ }^{16}$. Il est invité à exposer les propriétés de chacune des parties du discours (sauf l'interjection) : matière plutôt théorique qui dégage la nature et la fonction des classes de mots.

Proprium nominis est substantiam vel qualitatem vel quantitatem significare : et proprium est pronominis pro nomine poni proprio et certas significare personas. Verbi vero proprium est actionem vel passionem sive utrumque cum modis et temporibus significare. Proprium adverbii est cum verbo poni, et sino eo perfectam significationem non habere, ut : bene lego. Participii proprium est tempus habere et casus; ideo a quibusdam verbum casuale dicitur. Proprium conjunctionis est cum omnibus partibus modo praeposita, modo postposita jungi et conjungere partes. Proprium praepositionis est anteponi casualibus semper separatim. (Mag.) [PL 101, 858-859]

Après cette synopse des proprietates, le maître s'efface pour faire place à un échange presque continu entre Franco et Saxo. Ceux-ci font le tour des diverses partes orationis [cf. Annexe II]. Le magister n'intervient dans cette

13. Ce passage est basé à la fois sur le texte de Boèce et celui de Diomède $(\mathrm{H}$. Keil, Grammatici Latini I, p. 426); cf. L. HolTz, ici même.

14. Ce passage est basé sur diverses sources : pour la référence au bon usage, sur Audax (H. Keil, Grammatici Latini VII, p. 321) et pour les quatre fondements, sur des textes de Quintilien, d'Audax, de Charisius, de Diomède et de Marius Victorinus; cf. M. Roger, L'enseignement..., p. 324-326.

15. Chacune des " espèces ", sauf la vox, la littera, la syllaba et la pars font l'objet d'une définition (PL 101, 858).

16. Ideoque modum manualis libelli excedere vultis (Mag.) [PL 101, 858]; cf. H.W. FoRTGENS, "De paedagoog... ", p. 80-81. 
longue section que de façon sporadique; ses interventions répondent à une nette fonction didactique, à savoir celle de fournir, à côté de la définition grammaticale, une définition philosophique (dialectique et sémiotique) - aristotélicienne et transmise à travers le commentaire de Boèce sur le Peri Hermeneias -, des deux principales parties du discours, le nom et le verbe $^{17}$.

(I) Interrogemus tamen, o Franco, magistrum philosophicam definitionem nominis. (Sa.)

- Nomen est vox significativa secundum placitum, sine tempore, definitum aliquid significans in nominativo, cum est aut non est, ut : homo est, homo non est. In [aliis] casibus licet addas est vel non est, nihil tamen certum significat, si non apponas quid sit vel quid non sit. Ut : hominis est, hominis non est. Secundum placitum, id est compositionem singularum gentium sunt nomina composita, ut quod Latine dicis aurum, hoc Graece crusov dicitur. Una est substantia, sed diversa nomina ${ }^{18}$. (Mag.) [PL 101, 859]

(II) Magis interrogemus magistrum, quae sit verbi definitio secundum philosophiam. (Sa.)

- An habet, magister, juxta philosophiae rationem haec tam principalis pars definitionem propriam, veluti nomen [habet]? (DIS.)

- Habet subtilem definitionem et nobilem, et est hujusmodi : Verbum est vox significativa secundum placitum, cum tempora, definitum aliquid significans et accidens. (Mag.)

- Expone, pater, hanc nobis definitionem. (DIS.)

- Dum dico, lego, actum significo legentis : quem actum alia Graecus significat voce; et ideo verbum secundum placitum est : tempus que attrahit. Definitum, id est, certum aliquid significat : et accidens; accidit enim actio vel passio homini ${ }^{19}$. (Mag.) [PL 101, 874]

C'est là d'ailleurs la dernière apparition du magister dans le texte : Alcuin s'est borné à définir d'un point de vue philosophique les deux parties du discours qui suffisent à construire une phrase.

\section{La position " intertextuelle " du dialogue}

Il faut faire ici la distinction entre trois types de rapports intertextuels.

A- D'un côté, ce texte d'Alcuin se rattache à toute sa production, et tout particulièrement à ses autres écrits didactiques. Parmi ceux-ci, on retiendra, comme textes d'encadrement général, l'Epistola de litteris colendis et

17. Pour une analyse détaillée de l'emploi fait par Alcuin (dans son ars grammatica et sa Dialectica) du commentaire de Boèce, voir la contribution de L. Holtz, ici même; voir aussi M. Roger, L'enseignement..., p. 339-340 et H.-W. ForTGENS, « De paedagoog... ", p. 61-62.

18. Cf. Dialectica : C. Quid est, secundum placitum.-A. Id est, secundum compositionem singularum gentium. Nam res eaedem omnibus gentibus sunt; nomina vero diversa, et secundum placitum imponentium composita, ut auri eadem natura est apud Graecos et Latinos; dum illi hoc Kryson nominant, isti aurum [PL 101, 973].

19. Cf. Dialectica [PL 101, 974]. 
l'Admonitio generalis, et, comme textes à contenus doctrinaux partiellement ou ponctuellement convergents :

- le de orthographia (cf. les passages à propos de la signification de prépositions; l'usage des termes compositio et separatio à propos des éléments formatifs; le renvoi aux espèces grammaticales);

- le Dialogus de rhetorica et virtutibus;

- la Dialectica (cf. notions du proprium, de la notion ad aliquid, de la definitio, et définitions du nom, du verbe et de l'oratio);

- la Disputatio Pippini regalis et nobilissimi juvenis cum Albino scholastico (définition de la littera);

- la Disputatio de vera philosophia (qui dans beaucoup de manuscrits figure comme introduction au dialogue entre Franco et Saxo).

On n'oubliera surtout pas les extraits qu'Alcuin a réunis à partir de sa lecture attentive de Priscien. Ces Excerptiones super Priscianum ${ }^{20}$ témoignent d'une lecture intelligente des Institutiones. Les extraits s'étendent sur l'œuvre complète ${ }^{21}$, surtout les deux derniers livres de syntaxe (= Priscianus minor), alors que dans le dialogue entre Franco et Saxo, Alcuin ne vise guère que le niveau de la morphologie ${ }^{22}$.

B- En deuxième lieu, l'opuscule se rattache à l'intérêt d'Alcuin pour la littérature latine classique, Virgile en tête, suivi par Térence, Cicéron, Horace et Juvénal : Alcuin, le "Flaccus " carolingien ${ }^{23}$, se plaît à larder son dialogue de nombreuses citations littéraires.

C- En troisième lieu, le texte se rattache à un réseau de sources (grammaticales), qu'Alcuin utilise de façon synthétique et homogénéisante.

\section{Traits caractéristiques de la démarche d'Alcuin}

Pour analyser la démarche d'Alcuin, on adoptera une double perspective.

\section{Alcuin et les doctrines grammaticales}

Alcuin propose une synthèse du savoir grammatical, qu'il enrichit et approfondit par une visée philosophique ${ }^{24}$. Il ne faut pas juger son texte

20. Sur ces extraits, voir J. R. O’Donnell, "Alcuin's Priscian » et L. Holtz, "Priscien dans la pédagogie... ". Sur la place de la syntaxe dans la grammaire carolingienne, voir A. LuHTALA, "Syntax and Dialectic...".

21. Les extraits proviennent des livres II-III, VIII, et XI à XVIII des Institutiones grammaticae.

22. En fait, cette dernière affirmation doit être nuancée : non seulement, le traitement des conjonctions empiète sur la syntaxe phrastique, mais il y a aussi le bref aperçu des proprietates des parties du discours qui dégage des aspects fonctionnels, au-delà des traits morphologiques.

23. Voir L. HoLTz, "Alcuin et la réception de Virgile... ", p. 74-75.

24. Voir H.-W. FoRTGENS, " De paedagoog... ", p. 61 ; E. VINEIS, " Grammatica e filosofia... "; M. IRvine, The Making of Textual Culture..., p. 321-323; V. LAW, Grammar and Grammarians..., p. 138; P. Swiggers, Histoire de la pensée linguistique..., p. 95; et le texte de L. HoLTz, ici même. 
avec des yeux modern(ist)es : ni le concept de "propriété littéraire/intellectuelle " ou d'» appropriation ", ni celui d'» originalité " sont de mise ici, dans une culture où l'apprentissage oral, basé sur la voix d'autorité, et l'insertion dans la tradition sont la norme. Alcuin est, si l'on veut, hautement " original " par la combinatoire qu'il met en place et qu'il livre au lecteur dans un style soutenu et dans une forme agréable. Il est également " original " par la réussite avec laquelle il passe de l'information caractéristique du manuel grammatical (en récupérant aussi les listes à orientation lexicale d'ouvrages comme celui de Phocas) ${ }^{25}$ à une information de niveau supérieur (" magistral ").

Cet effort de synthétiser le savoir grammatical de base en l'augmentant d'une vue d'en haut, se traduit dans l'intégration de diverses sources. Alcuin utilise Donat, Priscien, Phocas (pour le traitement des declinationes en fonction des lettres finales), Audax, Diomède, Pompéius (à un ou deux endroits) ${ }^{26}$, Marius Victorinus, Cassiodore, Isidore de Séville, Bède (métrique) et, comme on l'a vu, Boèce (commentaire du Peri Hermeneias et intégration de l'Isagôge de Porphyre). Si Isidore de Séville est la source directe pour les species de la grammaire, les deux auteurs auxquels Alcuin renvoie de façon explicite, et qui sont aussi ses principales sources ${ }^{27}$, sont Donat et Priscien. [cf. Annexe III].

La macro-structure ainsi que la forme dialoguée proviennent de Donat : l'ordre de traitement des parties du discours (Nom - Pronom - Verbe Adverbe - Participe - Conjonction - Préposition - Interjection) est celui qu'on trouve chez Donat (et qui diffère de l'ordre " grec " adopté par Priscien ${ }^{28}$ ).

25. Phocas, Ars de nomine et verbo (H. Keil, Grammatici Latini V, p. 410-439). Ce que V. LAW, Grammar and Grammarians..., p. 130-131, appelle les "regulae grammars ", en opposition avec le type de la Schulgrammatik. La désignation des deux types est un peu malheureuse : il s'agit dans les deux cas d'ouvrages scolaires et les grammaires de type regulae sont en fait des grammaires-lexiques (regulae renvoie aux principes par lesquels on peut rattacher des formes lexicales à un paradigme ou sous-paradigme, en fonction d'un repère formel, le plus souvent la dernière lettre ou syllabe d'un mot).

26. L'influence de Pompéius pourrait concerner l'introduction de la disputatio : cf. Pompée (H. Keil, Grammatici Latini V, p. 96) : Multi ab litteris inchoant; nec hoc verum est. Multi et a voce inchoant ea ratione, quoniam litterae de voce sunt natae [...] nec hoc verum est, sed qui inchoat artem, debet inchoare ab octo partibus orationis. qua ratione? quia qui artem istam scribit, grammaticus dicitur. [...] de voce tractare quid est? hoc philosophorum est. de litteris tractare omnium est. [...] de partibus tractare, hoc grammatici est. Elle est plus nette dans le traitement des verba neutra, où Alcuin adopte la position de Pompéius (les verba neutra ont soit une valeur active soit une valeur passive; cf. H. Keil, Grammatici Latini V, p. 227, l. 35). Sur l'œuvre de Pompéius, voir L. Holtz, "Tradition et diffusion..." ".

27. Déjà chez Aldhelm, Donat et Priscien étaient respectivement illustris grammaticae artis commentator et Romanae lumen facundiae; cf. M. ROGER, L'enseignement..., p. 328 (n. 4 et 5 ).

28. Il s'agit de l'ordre suivant : NOM - VERBE - PARTICIPE - PRONOM - PREPOSITION - AdVERBE - INTERJECTION - CONJONCTION. La seule différence avec l'ordre des tekhnai grecques réside dans la disparition de l'article et dans le dédoublement de l'adverbe (en adverbium et interiectio). Voir à ce propos P. SWIGGERs, Histoire de la pensée linguistique..., p. 87-88. 
Pour les définitions - à part les définitions philosophiques du nomen et du verbum - Alcuin reprend en général Priscien (cela de façon très nette pour la définition de l'adverbium). C'est Priscien aussi qu'Alcuin suit dans sa conception restrictive du pronomen (comme signifiant des " personnes finies").

Les renvois explicites au nom propre Donatus $(=13)$ sont plus nombreux que ceux au nom de Priscianus (=6). Mais Alcuin accorde plus de poids à l'opinion de Priscien :

1. Après avoir énuméré les six accidents que Donat attribue au nom (Qualitas, Comparatio, Genus, Numerus, Figura, Casus), Saxo fait remarquer que Priscien n'en compte que cinq, puisqu'il réunit la qualitas et la comparatio sous la species. Or, c'est ce dernier terme qui est retenu dans la suite.

2. Dans le chapitre des pronoms, Alcuin reconnaît, tout comme Priscien - Latinae eloquentiae decus (Sa.) [PL 101, 873] -, seulement quinze pronoms (8 primitiva, 7 derivativa); les formes adjectivales quis, qualis, talis etc. que Donat avait incluses dans les pronoms, ne sont pas explicitement reconnues comme pronoms; Alcuin reproduit le raisonnement de Priscien :

Memor esto, dixisse te quindecim pronomina esse. Sed quid est quod Donatus inter pronomina posuit, quis, qualis, talis, quot, tot, quotus, totus [quantus, tantus]? (Fr.)

- Memini me dixisse quindecim esse pronomina, in quibus nulla dubitatio esset. De istis enim quae ponis, dubitatio est an sint pronomina, an nomina. Priscianus Latinae eloquentiae decus nomina interrogativa vel relativa vel redditiva ea omnino dicit, et pronomina negat esse posse, quia finitas personas non habent, quod proprium est pronominis cum casu juncti : sicut et alia novem nomina mobilia quidam errore seducti pronomina dicunt esse, quia pronominum declinationem habent, id est, unus, ullus, nullus, solus, totus, alius, alter, uter, neuter: quos multae lectionis ratio revincit. (Sa.) [PL 101, 873]

3. Le supin et le gérondif sont traités comme des noms, conformément à l'enseignement de Priscien.

4. À deux reprises, Alcuin reproche à Donat d'être trop succinct, trop vague et trop lacunaire.

Sed quia defectiva esse verba introduxisti, subito defectiva per singula accidentia opus habeo ut pandas mihi, maxime quia Donatus magister noster haec valde obscure et breviter tetigit. (Fr.) [PL 101, 882 ${ }^{29}$

Quid a me quaeris, si tibi Donatus non sufficit? (Sa.)

- Non a te tanta quaesissem, si Donatus mihi suffecisset. (Fr.) [PL 101, 900]

À cette "supériorité " de Priscien, il y a une exception : dans la question des espèces de conjonctions, Alcuin se montre d'accord avec l'inventaire plus réduit de Donat, qui ne reconnaît que cinq types sémantiques.

29. L'enseignement de Donat est complété aussitôt après par celui de Priscien. 
Species, quas Donatus potestatem nominat, secundum eum quinque sunt : copulativa, expletiva, causalis, disjunctiva, rationalis. Secundum Priscianum plures. Sed hae quinque species principales sunt, aliasque in se continentes species [...] (Sa.) [PL 101, 895]

Hae et aliae species in Prisciano reperiuntur, quae tamen incidere possunt in praedictas quinque (Sa.) [PL 101, 896]

\section{Les doctrines grammaticales d'Alcuin}

Les doctrines grammaticales d'Alcuin constituent une dimension à plusieurs plans d'incidence. En effet, d'une part, il y a Alcuin auteur de cette disputatio centrée autour de la morphologie, et il y a Alcuin excerpteur (des passages syntaxiques) de Priscien.

D'autre part, il y a le grammairien qui synthétise l'enseignement grammatical, et à côté de lui, le grammairien qui prend de la hauteur, en proposant des définitions philosophiques du nom et du verbe.

En troisième lieu, il y a la doctrine grammaticale enseignée à travers les définitions (grammaticales) des parties du discours et il y a celle, légèrement ou sensiblement différente, qui est contenue dans le bref paragraphe sur les proprietates. Dans ce dernier cas, l'accent est en général mis sur le sémantisme de la classe grammaticale en question et sur des traits syntaxiques/syntactiques; qu'on compare respectivement les exposés sur les proprietates avec les définitions de l'adverbe (1a, 1b), du participe (2a, 2b), de la conjonction (3a, 3b) et de la préposition (4a, 4b) :

(1a) Proprium adverbii est cum verbo poni, et sino eo perfectam significationem non habere, ut : bene lego. (Mag.) [PL 101, 859]

(1b) Adverbium est pars orationis semper verbo cohaerens; et hoc agit verbis additum, quod adjectiva nomina appellativis adjuncta : ut, prudens homo prudenter agit, felix vir feliciter vivit. (Sa.) [PL 101, 886]

(2a) Participii proprium est tempus habere et casus; ideo a quibusdam verbum casuale dicitur. (Mag.) [PL 101, 859]

(2b) Participium dictum est, quia partem nominis partemque verbi tenet, et medium inter ea locum habet, et semper in derivatione est; quod nulla alia pars orationis habet, dum aliae partes primitivas habent species, unde derivantur. (Sa.) [PL 101, 889]

(3a) Proprium conjunctionis est cum omnibus partibus modo praeposita, modo postposita jungi et conjungere partes. (Mag.) [PL 101, 859]

(3b) Conjunctio est pars orationis indeclinabilis, conjunctiva et significativa, aliarum partium vim et ordinationem demonstrans. (Sa.) [PL 101, 895]

(4a) Proprium praepositionis est anteponi casualibus semper separatim. (Mag.) [PL 101, 859]

(4b) Pars orationis indeclinabilis. [Quid habet officii?] Ut aliis praeponatur partibus vel appositione, vel compositione. (Sa.) [PL 101, 896] 
Alcuin ne se limite pas à la classe lexico-grammaticale : il s'intéresse à une problématique morphosyntaxique, celle du statut de formes comme parties du discours, et par conséquent à l'argumentation concernant leur statut.

Quomodo discerni potest utrum nomina sint sic composita, an adverbia? (Fr.)

- Cum nomina loco adverbiorum accipiuntur, manent indeclinabilia, licet diversis jungantur casibus, ut, sublime volans, sublime volantis, sublime volanti, etc. Si vero nomina sunt, declinantur, ut, hoc sublime volans, hujus sublimis volantis, huic sublimi volanti, etc. (Sa.) [PL 101, 886 $]^{30}$

\section{L'apport scientifique et culturel d'Alcuin grammairien}

Comment apprécier l'apport scientifique d'Alcuin grammairien? Il y a presque un siècle, Wilhelm Schmitz, à la suite de J. Frey ${ }^{31}$, ne voyait dans le dialogue entre Saxo et Franco qu'un texte sans valeur intrinsèque et " arbitrairement compilé ${ }^{32}$ ".

Or, Alcuin est loin d'être un simple compilateur : d'une part, il combine de façon intelligente ses sources (en les confrontant) et il illustre ses énoncés avec des exemples littéraires qui révèlent sa grande culture littéraire. D'autre part, à plusieurs reprises Alcuin va au-delà d'une simple utilisation de ses sources grammaticales. Je me limiterai à trois exemples :

1-Si on compare la liste des significationes de l'adverbe chez Alcuin [cf. Annexe IV] avec les listes des grammairiens antiques, on constate que sa liste n'est identique avec aucune autre ${ }^{33}$. Il se rapproche le plus de Priscien, mais on notera qu'Alcuin :

(a) rejette par exemple le terme idiosyncrasique de abnegativa;

(b) adopte un autre ordre de présentation (sa séquence est plus cohérente du point de vue sémantique que celle de Priscien);

(c) ne commet pas l'incohérence qu'on peut reprocher à Priscien (à savoir d'ajouter des significationes en dehors de la liste énumérative).

30. Cf. Priscien (H. Keil, Grammatici Latini III, p. 65). Exemple comparable : Quid tum interest inter numerorum nomina indeclinabilia et inter adverbia? (Fr.) - Quod adverbia et singularibus et pluralibus similiter junguntur. Ut : millies dixi, et millies diximus. Nomina vero non nisi pluralibus, ut, mille homines diximus. (Sa.) [PL 101, 867].

31. J. FREY, De Alcuini arte grammatica commentatio.

32. Cf. W. Schmitz, Alcuins ars grammatica..., p. 85 : "Alcuins Arbeit ist, rein in wissenschaftlich grammatischer Hinsicht, ohne inneren Wert. Allein die äussere Form der Darstellung, der geschickte und natürliche Dialog, ist des Verfassers Eigentum. Inhaltlich stellt sich die ars als weitgehendste Entlehnung und Zusammenstellung aus verschiedenen Quellen dar. Der Vergleich mit diesen Quellen ergibt keinen neuen Gedanken und keinen wissenschaftlichen Fortschritt, ja in dem zusammengetragenen Stoffe nicht einmal eine bessere, durchdachte Ordnung, sondern mehr willkürliche Kompilation. " Pour une vue opposée, voir M. ROGER, L'enseignement..., p. 340-341. [Pour un relevé des jugements positifs et négatifs, voir H.-W. FonTGENS, " De paedagoog... ", p. 64.]

33. Voir l'analyse de la terminologie et de la classification des adverbes dans P. SWIGGERS et A. WOUTERs, "De adverbio..." . 
2- Dans le passage concernant les verba neutra (où Alcuin se rapproche le plus de Diomède et de Pompéius), on trouve une présentation beaucoup plus nette des verbes neutres que chez n'importe quel grammairien de l'Antiquité.

3- Enfin, dans sa définition du pronom, Alcuin se distingue des grammairiens de l'Antiquité par l'emploi de la formule nominis vice (posita/positum) et par l'intégration dans la définition de l'économie réalisée par l'emploi d'un pronom.

De plus, il importe de ne pas être dupe d'une application trop servile de la Quellenkritik; s'il est vrai qu'Alcuin puise aux textes de Donat, Priscien, Boèce, Cassiodore, Isidore de Séville, on devra aussi reconnaître qu'il a intégré l'enseignement de Priscien au dispositif donatien ${ }^{34}$ et qu'il a opéré la transition vers une approche grammaticale réfléchie, allant au-delà de l'enseignement formaliste. Le magister renoue, à travers Boèce, avec une approche philosophique qui était aux fondements des textes grecs dont Priscien s'inspirait.

La disputatio entre Franco et Saxo est un document à haute importance culturelle. D'abord, par le choix des interlocuteurs et de leurs rôles respectifs, ce texte est le reflet littéraire du parcours intellectuel et professionnel d'Alcuin. L'importation du savoir grammatical et « libéral » insulaire dans le monde franc est illustrée de façon concrète et ludique par l'instruction que fournit Saxo à Franco et par l'autorité scientifique du magister Albinus. La Renaissance carolingienne repose sur un enseignement systématique des artes liberales ${ }^{35}$, dont la grammaire (et la connaissance du latin classique) est la base ${ }^{36}$; la disputatio nous donne une bonne idée de la façon dont la matière grammaticale était enseignée et répétée dans un contexte didactique où l'oralité et l'exercice mémoriel étaient fondamentaux.

Le grand mérite d'Alcuin réside dans la synthèse intelligente du savoir grammatical antique, synthèse qui se voit enrichie d'une dimension philosophique : une réflexion sur la fonction du langage comme ensemble de symboles, sur la conventionnalité des signes linguistiques et sur le sémantisme des parties du discours. Alcuin annonce par là l'orientation philosophique que prendra la grammaire dès la seconde moitié du XII ${ }^{\mathrm{e}}$ siècle.

34. La synthèse de Donat et de Priscien réalisée par Alcuin est nettement plus réussie que celles de Donat et de Consentius (chez Tatwin) et de Donat et Charisius (chez Boniface); cf. M. Roger, L'enseignement..., p. 332-336. Pour une comparaison succincte de la grammaire d'Alcuin avec celles de Smaragde et de Raban Maur, voir H.-W. FoRTGENS, «De paedagoog... », p. 64-65.

35. Sur le rôle d'Alcuin dans le " programme " carolingien de renaissance des arts libéraux, voir W. EDELSTEIN, Eruditio... et L. HoLTZ, " Alcuin et la renaissance des arts libéraux ".

36. Sur le rôle crucial de la grammaire dans la culture médiévale, voir M. IRvinE, The Making of Textual Culture... (à propos d'Alcuin, voir p. 313-326). 


\section{Annexe 1 - Les species/divisiones de la grammaire}

Isidore

Divisiones autem grammaticae artis a quibusdam triginta dinumerantur, id est

partes orationis octo

vox articulata
littera
syllaba

pedes
accentus
positurae
notae
orthographia
analogia
etymologia
glossae
differentiae
barbarismi
soloecismi
vitia
metaplasmi
schemata
tropi
prosae
metra
fabulae
historiae

Alcuin

[Grammatica dividitur] In XXVI [species]

\author{
In vocem \\ in litteras \\ in syllabas \\ partes \\ dictiones \\ orationes \\ definitiones \\ pedes \\ accentus \\ posituras \\ notas \\ orthographiam \\ analogiam \\ etymologiam \\ glossas \\ differentias \\ barbarismum \\ soloecismum \\ vitia \\ metaplasmum \\ schemata \\ tropos \\ prosam \\ metra \\ fabulas \\ historias
}

\section{Annexe 2-Structure du dialogue}

[Préambule

les trois participants

l'ouverture de la disputatio]

littera

vox

divisio litterarum : vocales

consonantes

syllaba

grammatica

proprietates partium

de nomine

$$
\text { definitiones }
$$

accidentia 
Alcuin et les doctrines grammaticales

$\begin{array}{lll} & \text { qualitas } & \text { species } \\ & \text { comparatio } \\ & \text { genus } & \\ \text { numerus } & \\ \text { figura } & \text { casus } & \end{array}$

de verbo

definitio

accidentia

$$
\begin{aligned}
& \text { species } \\
& \text { persona } \\
& \text { figura* } \\
& \text { genus } \\
& \text { numerus } \\
& \text { casus }
\end{aligned}
$$

\section{definitiones} accidentia

significatio (= genus)
tempus
modus
species
figura
coniugatio**
persona
numerus

de adverbio

definitio

accidentia

\section{species}

figura*

significatio

de participio

\section{definitio}

accidentia

\section{genus}

casus

tempus

significatio*

numerus

figura

de coniunctione

\section{definitio}

accidentia

$$
\begin{aligned}
& \text { figura } \\
& \text { species } \\
& \text { ordo }
\end{aligned}
$$

de praepositione

definitio

$$
\text { (positio) }
$$

(significatio)

de interiectione

definitio

$$
\begin{aligned}
& \text { (significatio) } \\
& \text { (accentus) }
\end{aligned}
$$

* signale que l'accident est mentionné à un autre endroit dans l'énumération des accidents (au début de la section consacrée à une partie du discours).

** signale que l'accident n'est pas inclus dans l'énumération des accidents (au début de la section consacrée à une partie du discours).

() signale qu'on peut considérer le trait en question comme un accident, sans qu'Alcuin le dise explicitement [il n'y a pas de liste d'accidents pour la préposition et pour l'interjection]. 


\section{Annexe 3-Mentions de Donat [D] et de Priscien [P]}

PL 101, 855 D (accidentia litterarum)

PL 101, 857 D

PL 101, 859 D (accidentia nominis)

$859 \mathrm{P}$ (accidentia nominis)

PL 101, 860 D (aliae species nominis)

PL 101, 867 D (nomina semper singularia)

PL 101, 873 D

$873 \mathrm{P}$

PL 101, 877 D (species verbi) 2x

PL 101, 877 P (supina; participialia)

PL 101, 880 P (gerundiva)

PL 101, 882 D (verba defectiva)

PL 101, 895 D (species coniunctionis)

$895 \mathrm{P}$ (species coniunctionis)

PL 101, 896 P (species coniunctionis)

PL 101, 900 D (praepositio + casus)

PL 101, 900 D 2x

Note : PL 101, 857 : simple renvoi à Donatus; PL 101, 900 : les deux dernières mentions de Donat concernent l'information insuffisante qu'il fournit.

\section{Annexes 4 - Types de significationes des adverbes chez Alcuin}

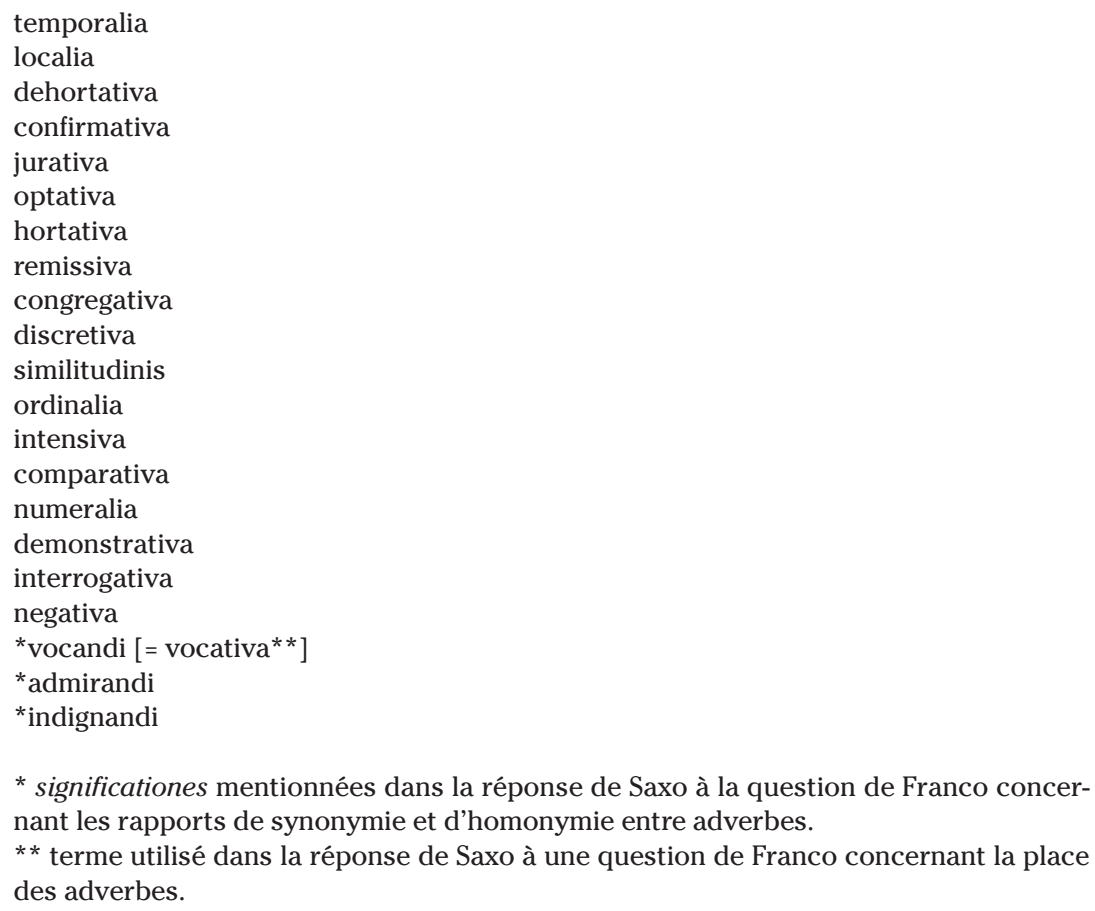

* significationes mentionnées dans la réponse de Saxo à la question de Franco concernant les rapports de synonymie et d'homonymie entre adverbes.

* * terme utilisé dans la réponse de Saxo à une question de Franco concernant la place des adverbes. 


\section{RESUME}

L'activité d'Alcuin dans le domaine grammatical constitue une pierre angulaire dans le renouveau des études "libérales " à l'époque carolingienne. Par son ars grammatica (= Dialogus Saxonis et Franconis de octo partibus orationis) et par ses Excerptiones super Priscianum, Alcuin a assuré la continuité des doctrines grammaticales gréco-latines en y ajoutant une dimension philosophique (récupération de l'enseignement aristotélicien, à travers Boèce). L'exposé fournit une analyse de la construction du discours didactique dans le dialogue entre Franco et Saxo, de la position " intertextuelle " de cet opuscule, des traits caractéristiques de la démarche d'Alcuin grammairien; il se termine par une évaluation de l'apport scientifique et culturel de l'œuvre grammaticale d'Alcuin.

\section{ABSTRACT}

Alcuin's activity in the field of grammar lies at the heart of the Carolingian renewal of the liberal arts. With his ars grammatica (= Dialogus Saxonis et Franconis de octo partibus orationis) and his Excerptiones super Priscianum, Alcuin was instrumental in realizing the continuous transmission of Greco-Latin grammatical conceptions, adding to these an important philosophical dimension (reintegration of Aristotelian ideas, through the translation and commentary of Boethius). This article offers an analysis of the build-up of Alcuin's didactical discourse in the dialogue between Franco and Saxo, of the intertextual position of the text, and of the characteristic features of Alcuin's grammatical approach; finally, a short evaluation of the scientific and cultural significance of Alcuin's grammatical work is given. 
